\title{
Chylous Ascites as a Complication of Infra-hepatic Inferior Vena Cava Tumor Resection
}

\author{
Gerald JS Tan* \\ Faculty of Medical Sciences, Newcastle University, UK
}

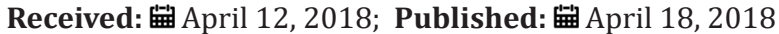

*Corresponding author: Gerald JS Tan, Faculty of Medical Sciences, The Medical School, Framlington Place, Newcastle University, Newcastle Upon Tyne NE2 4HH, UK

\begin{abstract}
This case study reports a case of an inferior vena cava leiomyosarcoma, where the patient had underwent resection of the tumour with reconstruction of the inferior vena cava and bilateral renal vein using a graft. At postoperative day 10 , the patient was noted to have a large amount of milky discharge from the laparotomy wound. The diagnosis of chyle leak was confirmed by fluid analysis that demonstrated high triglyceride content. Computed tomography (CT) of the abdomen revealed perihepatic collection, which was connected to the subcutaneous region. Aspiration under ultrasound guidance was performed for both the perihepatic and subcutaneous collection.
\end{abstract}

Keywords: Chylous ascites; IVC tumor resection; Chyle leak management

Abbreviations: CA: Chylous Ascites; IVC: Inferior Vena Cava; TG: Triglyceride; MCT: Medium-Chain Triglycerides; TPN: Total Parenteral Nutrition

\section{Introduction}

Chylous ascites is defined as a pathologic accumulation of chyle in the peritoneal cavity. The incident of chylous ascites (CA) following inferior vena cava (IVC) tumour resection is rare. There was only 1 incident of CA reported in 20,000 admissions at a large universitybased hospital over a 20-year period [1]. The development of CA is due to either direct injury or adhesion or extrinsic compression of lymphatic vessels. 1.1\% incident of CA post-abdominal surgery was reported, which includes inferior vena cava resection1. Progressive and painless abdominal distension is the commonest presenting complaint in CA [1-3]. Typically, high triglyceride ( $\mathrm{Tg}$ ) levels of more than $200 \mathrm{mg} / \mathrm{dL}$ is vital to differentiate between CA and pseudochylous ascites due to cellular degeneration from infection or malignancy [2]. The diagnostic approach of CA includes a careful history, physical examination, and diagnostic paracentesis [1]. These are vital for initial evaluation of patients who present with ascites. This case study reports a case of postoperative chylous ascites that presented with a milky-fluid discharged from the laparotomy wound on postoperative day 10 . The treatment options in managing such a case will also be discussed.

\section{Case Description}

A 64-year-old Chinese female underwent laparotomy and resection of an IVC leiomyosarcoma. Intra-operatively, there was a huge IVC tumor measuring around $7 \mathrm{~cm} \mathrm{x7} \mathrm{cm} \mathrm{x} 6 \mathrm{~cm}$, adhered densely with surrounding structures. The tumor was resected en-bloc and a venous graft was placed between proximal IVC and bilateral renal veins. The operation involved quit extensive adhesiolysis and resection. Post-operatively patient was monitored in the intensive care unit with a stable condition and was later transferred out on post-operative day 3. On postoperative day 10 , it was noted there was a serious discharge from the laparatomy wound but the patient had no complaints of abdominal distention or abdominal pain. Due to persistent serous discharge from the wound, sutures were removed over the proximal part of the wound, revealing large amounts of milky fluid leaking out. A stoma bag was applied at that area which had collected $647 \mathrm{cc}$ of milky fluid over the first 24 hours. The diagnosis of chyle ascites was made and was confirmed by fluid analysis, which demonstrated high content of triglycerides. 
CT abdomen revealed a collection of fluid deep to the subcutaneous tissue below the laparotomy wound with connection into the perihepatic collection inside the peritoneum. Ultrasound guided paracentesis of the fluid was performed on the next day and 60cc milky fluid mixed with blood was aspirated. Fluid was sent for analysis, culture and sensitivity and microbiology assessment. Patient remains a febrile and the stoma bag charting showed a reducing trend of fluid amount from 647 to $157 \mathrm{cc}$ per day. The patient was discharged home with the stoma bag to return in a week for follow-up. Upon review in clinic, there was no milky discharge observed from the wound. The patient reported it had stopped since postoperative day 17. Repeated Tran's abdominal ultrasound showed no ore perihepatic collection and the subcutaneous collection had also reduced in size. The patient was referred to the oncology team for further management (Figure 1).

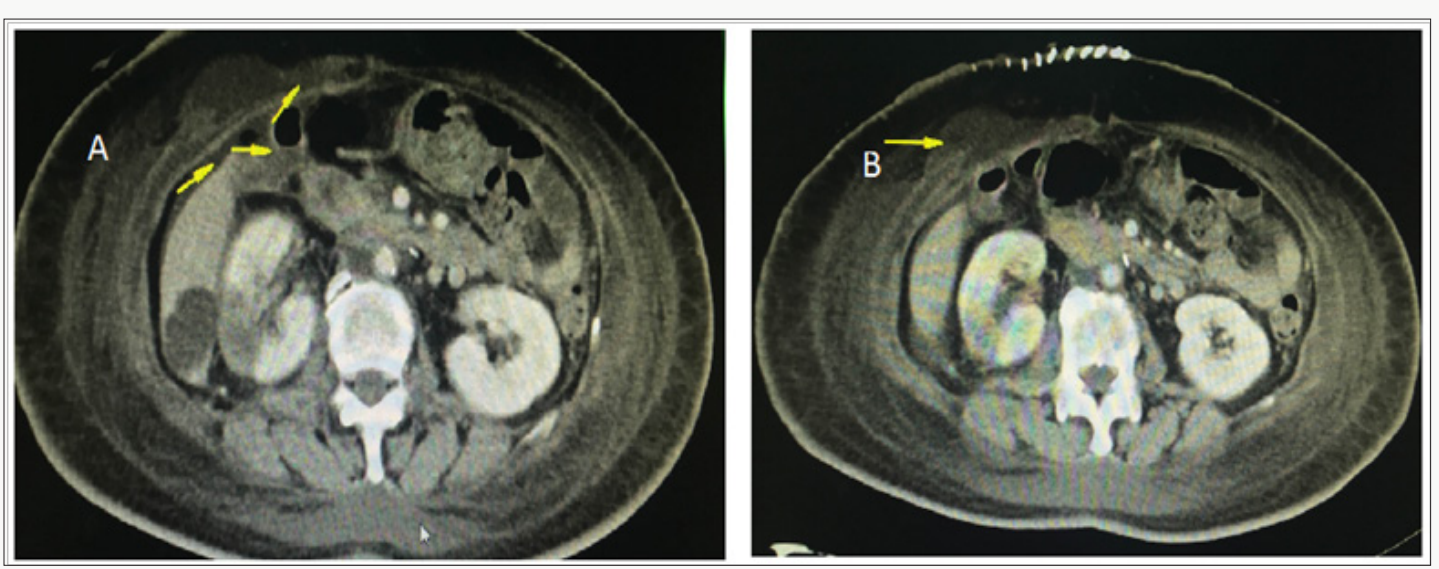

Figure 1: CT abdomen image shows area of perihepatic fluid collection (arrows) which was connected with subcutaneous area(a) An arrow in CT image (b) shows a subcutaneous fluid collection.

\section{Discussion}

In the case of IVC tumor resection, extensive dissection at the retroperitoneal space to achieve tumor clearance will cause disruption of cysterna chyli and subsequent formation of CA. Sudden interruption of venous return may also produce a high venous pressure caudad to the point of interruption, which would result in increase of lymphatic pressure and leaking of lymph into the peritoneal space $[1,2]$. Even though the incidence of postoperative CA is low, it can give high impact on patients' clinical conditions and recovery process. Most post-operative chyle leak can be successfully managed with conservative treatment including serial paracentesis, low-fat diet supplement with medium-chain triglycerides (MCT), total parenteral nutrition (TPN) and somatostatin [1,2]. Evans et al reported that conservative measures led to resolution rates of $65 \%$ in 23 patients within a median time of 13 weeks. The focus of conservative measures is to reduce the production and flow of the lymph and to re-store patients' nutrition balance and replace fluid and electrolytes $[3,4]$.

It was preferred to utilize low-fat diet with MCT in treating patient, which had demonstrated marked reduction in the amounts of chyle leakage. This approach was also combined with sandostatin administration to the patient. The use of sandostatin is justified as it showed successful results in treating lymph leakage after cardiac surgery, pelvic surgery and abdominal surgery [5-7]. It inhibits lymph secretion through somato statin receptor in the intestinal wall. The best result is achieved when it was used in combination with TPN or MCT diet [5,7].
Surgical intervention may be beneficial if conservative management failed to resolve the CA. Some suggest 2 weeks for surgical exploration to be performed if CA does not respond to conservative measures [1]. Aalami et al reported that $41 \%$ of their patients were treated successfully with direct surgical ligation of the leaking lymhatic gland. Moreover, surgical reintervations in postoperative CA carries high morbidity and mortality [1,3]. To overcome this problem, Konhoe et al described a combined method of pre and intra operative lymphangiography to help in identifying the site of lymhatic leakage. Through this measure, it can facilitate the successful surgical repair of the fistula. Postoperative lymphangiography with or without embolization is another promising technique that along with conservative measures, has the potential to avoid surgical re-intervention 8. Another surgical technique that has been proposed for the management of CA is peritoneovenous shunting. This technique has shown to successfully treated CA that are resistant to conservative therapy. Although this technique is less invasive, it carries high complication possibilities including sepsis, small bowel obstruction, electrolyte imbalance and occlusion of the shunt 2,5. Chyle drainage in the patient was stopped after 10 days of successful conservative treatment. Thus in this case, surgical intervention was not indicated [8].

\section{Conclusion}

There is no single best treatment in managing chylous ascites following resection of an IVC tumor. Various choices of conservative treatments had been used before and show favorable outcomes. Whenever possible, conservative measures should be attempted 
and considered adequately before the decision for invasive measures are undertaken.

\section{References}

1. Said A Al Busafi, Peter Ghali, Marc Deschênes, Philip Wong (2014) Chylous ascites: Evaluation and management. ISRN Hepatology, p. 10.

2. A Cárdenas, S Chopra (2002) Chylous ascites. American Journal of Gastroenterology 97(8): 1896-1900.

3. Talluri SK, Nuthakki H, Tadakamalla A, Jyothsna T, Siddesh B(2011) Chylous ascites. N Am J Med Sci 3: 438-440.

4. Laterre PF, Dugernier T, Reynaert MS (2000) Chylous ascites: diagnosis, causes and treatment. Acta Gastroenterol Belg 63 260-263.
5. Huang Q, Jiang ZW, Jiang J, Ning Li, Jie-Shou Li (2004) Chylous ascites: treated with total parenteral nutrition and somatostatin. World J Gastroenterol 10: 2588-2591.

6. A Smoke, MH Delegge (2008) Chyle leaks: consensus on management? Nutrition in Clinical Practice 23(5): 529-532.

7. AE Yildirim, R Altun, S Can, Ocal S, Akbas E, et al. (2011) Idiopathic chylous ascites treated with total parenteral nutrition and octreotide. A case report and review of the literature. European Journal of Gastroenterology and Hepatology 23(10): 961-963.

8. T Matsumoto, T Yamagami, T Kato, Hirota T, Yoshimatsu R, et al. (2009) The effectiveness of lymphangiography as a treatment method for various chyle leakages. British Journal of Radiology 82(976): 286-290.
(C) This work is licensed under Creative

To Submit Your Article Click Here:

Submit Article

DOI: 10.32474/OAJOM.2018.01.000114

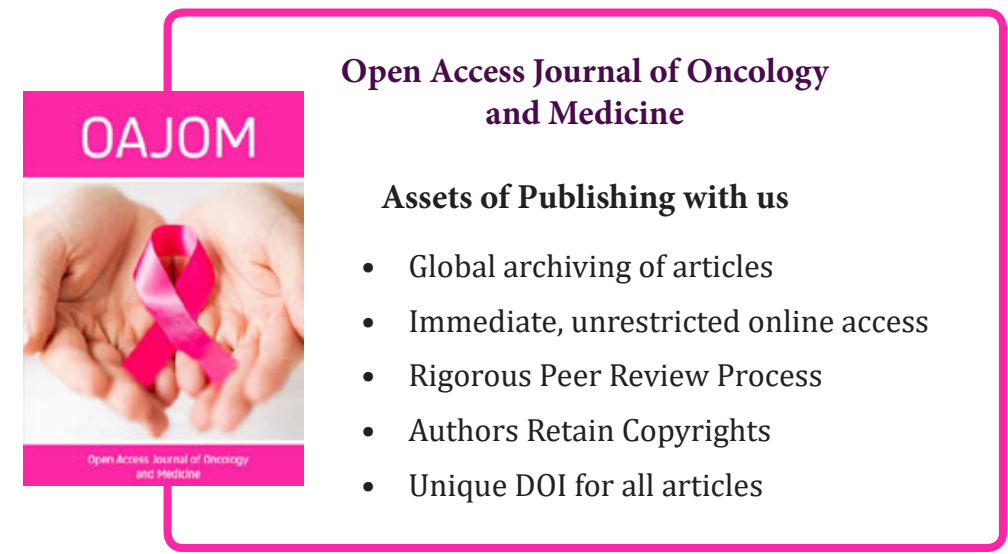

